\author{
J. L. Chan • V. Stoyneva $\cdot$ T. Kelesidis • \\ P. Raciti - C. S. Mantzoros
}

\title{
Peptide YY levels are decreased by fasting and elevated following caloric intake but are not regulated by leptin
}

Received: 7 July 2005 / Accepted: 21 August 2005 / Published online: 9 December 2005

(C) Springer-Verlag 2005

\begin{abstract}
Aims/hypothesis: Peptide YY (PYY) is a gutderived hormone that has been shown to reduce short-term food intake in animals and humans. It has been proposed that deficiency of PYY contributes to obesity in humans. However, the physiology of PYY regulation by factors such as caloric restriction, or by other molecules important in energy homeostasis, e.g. leptin, remains to be fully elucidated. Materials and methods: We evaluated the effect on PYY levels of: (1) caloric ingestion (a mixed meal) in five healthy normal-weight subjects; (2) fasting for 2 or 3 days in eight lean men and seven lean women respectively; and (3) recombinant human leptin administration at physiological replacement and pharmacological doses. Results: PYY levels increased $50 \%$ after a mixed meal $(p=0.01)$, and shortterm complete fasting for 2 or 3 days decreased leptin and PYY levels to $20-30 \%$ and $40-60 \%$ of baseline, respectively (both $p<0.05$ ). However, recombinant human leptin administration at physiological doses to restore the fasting-induced decrease of leptin levels and at pharmacological doses over the short term had no effect on PYY levels. Conclusions/interpretation: PYY increases after meal ingestion and decreases after fasting in a manner consistent with a meal-related signal of energy homeostasis. Importantly, circulating levels of this gut-secreted molecule are independent of regulation by leptin over the short term. These findings contribute towards our understanding of the homeostatic systems that regulate appetite in humans, including the possible redundancy of gastrointestinally secret-
\end{abstract}

\footnotetext{
J. L. Chan · V. Stoyneva - T. Kelesidis ·

P. Raciti · C. S. Mantzoros $(\bowtie)$

Division of Endocrinology,

Diabetes, and Metabolism,

Department of Medicine,

Beth Israel Deaconess Medical Center,

Harvard Medical School,

Boston, MA 02215, USA

e-mail: cmantzor@bidmc.harvard.edu

Tel.: +617-667-8630

Fax: +617-667-8634
}

ed and adipocyte-secreted signals. This may be of importance for the future development of medications to treat obesity.

Keywords Appetite · Fasting · Leptin - Obesity · Peptide YY

Abbreviations GCRC: General Clinical Research Center · PYY: Peptide YY · PYY 3-36: Peptide YY $3-36$

\section{Introduction}

Regulation of appetite and body weight requires the complex integration of signals (hormonal, metabolic and neural) deriving from several peripheral organs, including the gutderived peptide YY (PYY) and its cleaved product peptide $\mathrm{YY}_{3-36}\left(\mathrm{PYY}_{3-36}\right)$. $\mathrm{PYY}_{3-36}$ has been shown to reduce food intake over the short term in animals [1,2] and humans [3] by acting on neuropeptide $\mathrm{Y}$ receptors in the hypothalamus. In a recent study, obese subjects were found to have lower baseline PYY levels than lean subjects and were not resistant to the anorectic effect of exogenously administered $\mathrm{PYY}_{3-36}[3]$.

The physiology of PYY regulation and the potential

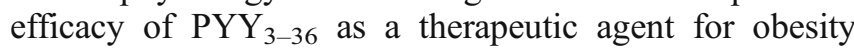
remain controversial, however, because recent extensive studies by independent groups of investigators have not been able to replicate the inhibitory effect of $\mathrm{PYY}_{3-36}$ on food intake in animals [4]. Moreover, the previously published data on PYY regulation in humans, including differences in PYY levels between lean and obese subjects [3] have not been replicated in a more recent study [5]. Finally, important questions remain unanswered, including whether PYY secretion is regulated by factors such as caloric restriction, i.e. through short-term complete fasting, or by other hormones important in energy homeostasis, such as the adipocyte-secreted leptin, which acts to reduce appetite through hypothalamic neuropeptide Y [6]. The question of whether PYY is altered by changes in leptin through 'crosstalk' with fat-derived signals of energy homeostasis has direct relevance for understanding the mechanism(s) by 
which appetite suppression occurs and thus potentially may have future therapeutic applications in the management of obesity.

We thus performed a series of interventional studies in healthy lean subjects to clarify PYY physiology in humans and to then investigate the relationship between the PYY and leptin pathways for regulating energy homeostasis. To study whether PYY levels respond to caloric administration or deprivation in the manner expected for a molecule that is important in energy homeostasis [3,7], we examined the effects of caloric administration (in the form of a standardised mixed meal) and of fasting on PYY levels. We then investigated the potential mechanisms underlying PYY regulation by changing leptin levels through a series of interventions. We sought to evaluate whether PYY is independent from the leptin system by investigating the effect of marked hypoleptinaemia (induced by complete fasting for either 2 or 3 days) on PYY levels. We then administered recombinant human leptin at physiological replacement doses during a similar fasted condition in the same subjects to test directly whether leptin has a role in regulating PYY levels. Finally, we investigated whether increasing leptin levels above the normal physiological range has a doseresponse effect on PYY. We did this by administering pharmacological doses of recombinant human leptin to healthy subjects.

\section{Subjects, materials and methods}

\section{Human subjects}

The study protocols were approved by the Institutional Review Board of the Beth Israel Deaconess Medical Center, and subjects gave written informed consent to participate. For the interventional studies involving recombinant human leptin administration, clinical quality recombinant human leptin was supplied by Amgen (Thousand Oaks, CA, USA) and administered under an Investigational New Drug application submitted to the Food and Drug Administration by the investigators (CSM).

Study 1: Effect of a mixed meal on PYY levels Five lean, healthy women were admitted to the General Clinical Research Center (GCRC) and studied twice in the fed state on a standardised isocaloric diet with $20 \%$ calories from breakfast (07:15 hours), 35\% from lunch (14:00 hours), $35 \%$ from dinner (18:00 hours), and 10\% from a snack (22:00 hours). Subjects were acclimatised to the GCRC by being admitted the night before the study day. Blood samples for measurement of PYY and leptin levels were obtained for each study before and $2 \mathrm{~h}$ after dinner (18:00 and 22:00, respectively) when leptin levels were not significantly different from fasting morning values, and the averages of the two measurements (one from each of the two studies) at each time point are reported here. The amount of energy ingested during dinner was $3129.7 \pm 76.7 \mathrm{~kJ}$.
Study 2: Effect of short-term fasting and/or fasting-induced hypoleptinaemia with and without administration of replacement-dose recombinant human leptin on PYY levels This study involved two groups of subjects. The first group comprised eight lean men, who were studied during three separate admissions in the GCRC as part of a larger study to evaluate the role of leptin in the neuroendocrine [8] and immune [9] responses to fasting. There was a baseline study on an isocaloric weight-maintaining diet $(n=8)$; a 2-day complete fasting study with administration of placebo $(n=8)$; and a similar fasting study with administration of replacement-dose recombinant human leptin designed to normalise the fasting-induced decline in leptin levels $(n=6)$. The same subjects participated in all three admissions, which were separated by at least 7 weeks, except for two subjects who did not complete the admission for fasting with recombinant human leptin administration. Subjects were admitted to the GCRC the night before the first study day, and during each study in the fed or fasting state, blood samples were obtained at 08:00 on days 1 and 3 for measurement of serum PYY and leptin levels. During the baseline study in the fed state, subjects received a standardised isocaloric diet: $20 \%$ calories from breakfast (08:00 hours), 35\% from lunch (14:00 hours), 35\% from dinner (18:00 hours), 10\% from a snack (22:00 hours). During both fasting studies, subjects received only caffeinefree and calorie-free liquids for 2 days and $\mathrm{NaCl}(500 \mathrm{mg})$, $\mathrm{KCl}(40 \mathrm{mEq})$, and a standard multivitamin with minerals daily. During one fasting study, recombinant human leptin was administered at a dose of $0.04 \mathrm{mg} \mathrm{kg}^{-1}$ day $^{-1}$ on the first day and $0.1 \mathrm{mg} \mathrm{kg}^{-1}$ day $^{-1}$ on the second and third days, a dosing regimen that was designed on the basis of prior pharmacokinetic studies to achieve physiological serum leptin levels similar to those in the fed state [10]. The total daily leptin dose for each day was divided into four equal doses given every $6 \mathrm{~h}$ by subcutaneous injection. During the other fasting study, placebo (a buffer solution) was administered at the same volume as the corresponding recombinant human leptin dose and according to the same schedule.

In the second group, seven lean women participated in a similar protocol (baseline state $[n=7]$, fasting with placebo administration $[n=6]$, fasting with replacement-dose recombinant human leptin administration [ $n=7]$, each separated by at least 8 weeks), except that the duration of fasting was 3 days, with blood samples obtained on days 1 and 4 of each admission. The replacement dose of recombinant human leptin was twice that administered to men, given the known sexual dimorphism of leptin levels with women having higher baseline leptin levels than men [6].

Study 3: Effect of physiological vs pharmacological doses of recombinant human leptin on PYY in the fed state The five lean women studied in the fed state during Study 1 were also studied within the context of a recombinant human leptin administration study. Subjects received a single physiological dose $(0.01 \mathrm{mg} / \mathrm{kg})$ of recombinant human leptin by subcutaneous injection at 08:00 hours during one study and a single pharmacological dose $(0.1 \mathrm{mg} / \mathrm{kg})$ at 
08:00 hours by subcutaneous injection during a second study day. Blood samples for PYY and leptin were obtained at 1,6 and $8 \mathrm{~h}$ after the dose of recombinant human leptin during each study (approximately $1.5 \mathrm{~h}$ after breakfast, prior to lunch, and $2 \mathrm{~h}$ after lunch, respectively).

\section{Measurements}

Leptin was measured by radioimmunoassay (Linco Research, St Charles, MO, USA) with sensitivity $0.5 \mathrm{ng} / \mathrm{ml}$, intra-assay CV 6-7\%. PYY was measured using an enzymelinked immunosorbent assay (Diagnostic Systems Laboratory, Inc., Webster, TX, USA) with sensitivity $3.5 \mathrm{pmol} / 1$, intra-assay CV $3.0 \%$, inter-assay CV $7.4 \%$, and $100 \%$ crossreactivity for the full-length peptide $\left(\mathrm{PYY}_{1-36}\right)$ and the truncated $\mathrm{PYY}_{3-36}$, both of which have biological activity.

\section{Statistical analysis}

Data are presented as mean \pm SEM. Non-parametric (Wilcoxon signed rank) tests were used to compare hormone levels before and after a meal (Study 1); between baseline (day 1) and post-intervention (day 3 or 4 ) in the fasting and leptin replacement study (Study 2); and between AUCs for leptin levels after physiological and pharmacological recombinant human leptin doses (calculated using the standard trapezoidal method) and between hormone levels at $+1,+6$, and $+8 \mathrm{~h}$ time points in Study 3 . Analyses were carried out using SPSS 11.5 (SPSS, Chicago, IL, USA).

\section{Results}

Effect of a mixed meal on PYY levels of lean subjects

We first sought to confirm the effect of a meal on PYY levels. In a group of lean women (age $=20.4 \pm 0.7$ years, $\mathrm{BMI}=21.9 \pm 0.7 \mathrm{~kg} / \mathrm{m}^{2}$ ) who received a standardised, isocaloric diet at fixed times during the day, serum PYY levels increased by approximately $50 \%$ from premeal $(18: 00)$ to $2 \mathrm{~h}$ postdinner ( $14.6 \pm 2.8 \mathrm{pmol} / \mathrm{l}$ to $22.9 \pm 5.3 \mathrm{pmol} / \mathrm{l}, p=0.01)$ (Fig. 1). Serum leptin levels before and after dinner were not significantly different from fasting morning levels (fasting: $15.0 \pm 3.0 \mathrm{ng} / \mathrm{ml}$ vs predinner $25.5 \pm 4.7 \mathrm{ng} / \mathrm{ml}, p=0.07$; fasting vs postdinner $19.9 \pm 3.4 \mathrm{ng} / \mathrm{ml}, p=0.24$ ).

Effect of short-term complete caloric restriction and/or fasting-induced decrease in circulating leptin on PYY levels

We then investigated whether changes in leptin levels induced by acute energy deficit (i.e. complete fasting for 48 to $72 \mathrm{~h}$ ) would regulate PYY levels and whether any fastingassociated changes in PYY occur through a leptin-dependent or -independent mechanism, using a paradigm of

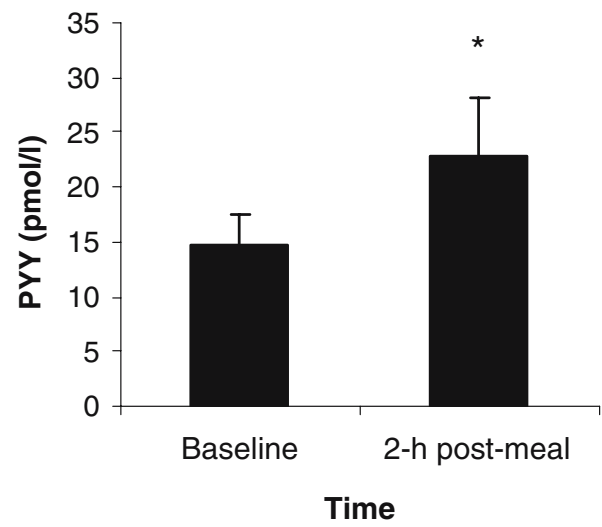

Fig. 1 Serum PYY levels in response to mixed meal ingestion. Serum PYY levels before (18:00 hours) and $2 \mathrm{~h}$ after (20:00 hours) ingestion of a standardised meal. $n=5$, with the average of two measurements per subject presented; ${ }^{*} p<0.05$ vs. baseline

studying the same subjects during complete fasting alone and complete fasting with leptin replacement. Subjects were first studied in the fed state over 2 days (men, age $=23.3 \pm$ 1.2 years) or 3 days (women, age $=22.4 \pm 1.2$ years), during which their BMI remained stable (men: $23.2 \pm 0.5$ to $23.3 \pm$ $0.5 \mathrm{~kg} / \mathrm{m}^{2}, p=0.58$ ) or increased slightly (women: $21.7 \pm 0.8$ to $22.1 \pm 0.7 \mathrm{~kg} / \mathrm{m}^{2}, p=0.02$ ). Similarly, leptin levels remained stable (men: $1.7 \pm 0.6$ to $1.7 \pm 0.3 \mathrm{ng} / \mathrm{ml}, p=0.26$ ) or increased slightly (women: $11.0 \pm 1.6$ to $16.2 \pm 1.3 \mathrm{ng} / \mathrm{ml}$, $p=0.03$ ). PYY levels remained unchanged (men: $29.9 \pm 7.2$ to $35.0 \pm 7.2 \mathrm{pmol} / 1, p=0.21$; women: $20.9 \pm 6.5$ to $15.6 \pm$ $3.5 \mathrm{pmol} / 1, p=0.18$ ).

Complete fasting for 2 or 3 days in lean men and women, respectively, resulted in a small but significant decrease in BMI (men: $23.4 \pm 0.6$ to $22.7 \pm 0.6 \mathrm{~kg} / \mathrm{m}^{2}, p=0.01$; women: $21.9 \pm 1.1$ to $21.4 \pm 1.1 \mathrm{~kg} / \mathrm{m}^{2}, p=0.03$ ). In response to this duration of fasting, serum leptin levels decreased markedly to approximately $20-30 \%$ of baseline (men: $2.1 \pm 0.6$ to $0.7 \pm$ $0.3 \mathrm{ng} / \mathrm{ml}, p=0.01$; women: $14.7 \pm 2.6$ to $2.8 \pm 0.3 \mathrm{ng} / \mathrm{ml}$, $p=0.03$ ), i.e. out of proportion to the change in BMI. PYY levels decreased to approximately $40-60 \%$ of baseline (men: $31.8 \pm 6.5$ to $18.6 \pm 6.6 \mathrm{pmol} / 1, p=0.03$ [Fig. 2a]; women: $16.3 \pm 7.9$ to $7.2 \pm 2.9 \mathrm{pmol} / \mathrm{l}, p=0.03$ [Fig. 2b]).

When recombinant human leptin was administered during fasting, despite a similar small decrease in BMI, serum leptin levels were fully restored to levels that were higher than baseline (men: $2.3 \pm 0.8$ to $4.9 \pm 0.8 \mathrm{ng} / \mathrm{ml}, p=0.03$; women: $12.2 \pm 1.6$ to $27.9 \pm 2.5 \mathrm{ng} / \mathrm{ml}, p=0.02$ ) but within the physiological range for lean subjects. Despite increasing serum leptin levels, however, recombinant human leptin administration during fasting did not alter the significant decline in PYY levels associated with fasting (men: $39.0 \pm$ 9.9 to $16.5 \pm 4.1 \mathrm{pmol} / 1, p=0.03$ [Fig. $2 \mathrm{a}$ ]; women: $32.4 \pm 8.4$ to $6.4 \pm 2.3 \mathrm{pmol} / 1, p=0.02$ [Fig. $2 \mathrm{~b}$ ]).

Serum leptin levels measured across time on the baseline day of each fed or fasting condition were significantly higher in women compared with men $(12.5 \pm 1.6$ vs $2.0 \pm$ $0.4 \mathrm{ng} / \mathrm{ml}$ ), consistent with the known sexual dimorphism of leptin levels. PYY levels measured at the same time points 
Fig. 2 Effect of fasting and recombinant human leptin replacement on PYY levels. Serum PYY levels in (a) lean men $(n=8)$ and (b) lean women $(n=7)$ at baseline (blue bars) and after 2-day (men) or 3-day (women) fasting (purple bars). Treatment was with placebo or with replacement-dose recombinant human leptin. ${ }^{*} p<0.05$ vs day 1 a

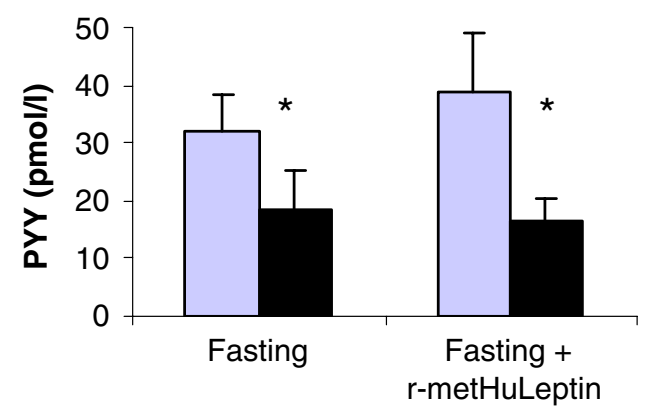

b

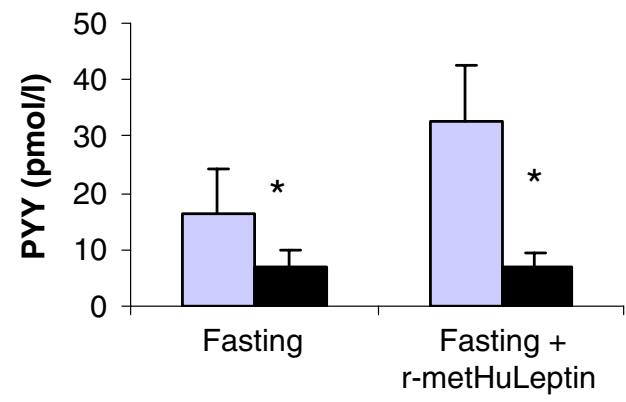

tended to be lower in women compared to men $(23.5 \pm 6.3 \mathrm{vs}$ $33.1 \pm 4.3 \mathrm{pmol} / \mathrm{l})$ although the difference did not reach significance $(p=0.06)$.

Effect of increasing circulating leptin on PYY levels: physiological vs pharmacological dose recombinant human leptin administration

Finally, since decreasing leptin levels and/or physiological replacement-dose recombinant human leptin had no effect on PYY levels, we investigated whether this may have been the result of a potential threshold phenomenon or a dose-response effect. We tested this by increasing leptin levels pharmacologically through administration of two separate doses of recombinant human leptin to lean subjects (a dose of $0.01 \mathrm{mg} / \mathrm{kg}$ to increase leptin levels within the physiological range, and a ten-fold higher dose of $0.1 \mathrm{mg} / \mathrm{kg}$ to increase leptin levels to pharmacological ranges).

As expected, the AUC for leptin levels was significantly higher after the pharmacological dose of recombinant human leptin than after the lower, physiological dose $(217.1 \pm 50.7$ vs $882.2 \pm 84.4, p=0.04)$. In contrast, there was no significant difference in AUC for PYY after the physiological or pharmacological doses of recombinant human leptin (707.8 130.5 vs $932.4 \pm 289.6, p=0.23$ ). Consistent with this finding, serum leptin levels were significantly higher at each time point after the pharmacological dose of recombinant human leptin than after the physiological dose ( $1 \mathrm{~h}: 110.3 \pm 5.6$ vs $24.5 \pm$ $4.5 \mathrm{ng} / \mathrm{ml} ; 6 \mathrm{~h}: 108.7 \pm 13.2$ vs $22.0 \pm 5.5 \mathrm{ng} / \mathrm{ml} ; 8 \mathrm{~h}: 64.4 \pm$ 10.0 vs $17.1 \pm 4.5 \mathrm{ng} / \mathrm{ml}$; all $p=0.04)$. PYY levels were not significantly different in response to the pharmacological as compared with the physiological dose of recombinant human leptin at the corresponding time points ( $1 \mathrm{~h}: 18.0 \pm$ 6.1 vs $16.1 \pm 2.9 \mathrm{pmol} / 1, p=0.89 ; 6 \mathrm{~h}: 18.0 \pm 7.7$ vs $14.1 \pm$ $2.7 \mathrm{pmol} /, p=0.89 ; 8 \mathrm{~h}: 24.2 \pm 4.8$ vs $15.4 \pm 3.2 \mathrm{pmol} / 1$, $p=0.14)$.

\section{Discussion}

In this study, we confirm that PYY levels increase in response to caloric ingestion in humans, as others have shown $[5,7,11]$, and demonstrate the novel finding that complete fasting for 48 to $72 \mathrm{~h}$ in healthy subjects significantly decreases PYY levels-findings that are consistent with the notion that PYY is an anorexigenic hormone that acts to reduce appetite. In addition, this study provides important, clinically relevant insights into potential interaction(s) of the gut and adipocyte hormone systems that regulate appetite and body weight by demonstrating that PYY is not regulated by short-term changes of leptin levels at physiological or pharmacological ranges.

PYY is secreted from gastrointestinal cells (mainly distal small intestine and colon) in levels that correspond to the amount of calories ingested [7]. The demonstration that exogenous $\mathrm{PYY}_{3-36}$ administration in rodents inhibits food intake and reduces weight gain via the neuropeptide Y2 receptor generated substantial scientific interest in the potential use of this hormone as a therapeutic agent for obesity [1]. These results were further supported by findings from the same investigators that infusion of $\mathrm{PYY}_{3-36}$ decreased caloric intake in both obese and lean humans by 26 and 33\%, respectively, over $24 \mathrm{~h}$ [3]. In contrast, since most obese humans are resistant to the adipocyte-secreted hormone leptin [12], which also acts on hypothalamic neurons (including neuropeptide Y neurons) to decrease food intake, exogenous leptin administration in obese subjects had only modest success in inducing weight loss [13].

The anorectic effect of $\mathrm{PYY}_{3-36}$ in humans has recently been questioned, however, since extensive efforts by independent groups have been unable to replicate the findings that $\mathrm{PYY}_{3-36}$ decreases food intake in rodents [4] In addition, it has been suggested that PYY deficiency may be important in the pathogenesis of obesity, since obese persons were shown to have lower PYY levels than lean persons [3]. However, this has not been supported by the work of other investigators [5]. Thus, the physiological regulation of PYY in humans remains poorly understood. We thus performed studies to investigate whether PYY is regulated by acute changes in nutritional status, first confirming that PYY levels increase significantly after caloric ingestion (in the form of a mixed meal) using an assay different from previously published assays $[5,7,11]$.

We demonstrate for the first time herein that PYY levels are decreased by complete fasting for 48 to $72 \mathrm{~h}$ in lean subjects, a finding that is not unexpected if PYY functions as an anorectic hormone. Unlike other hormonal axes [8], however, the fasting-induced decrease in PYY levels is not mediated by falling leptin levels since restoration of leptin 
levels with exogenous recombinant human leptin administration does not alter the decline in PYY that is associated with acute energy deprivation. Moreover, exogenous administration of recombinant human leptin in doses sufficient to achieve physiological and pharmacological leptin levels does not regulate PYY in the short term, suggesting that, similar to our previous findings on the lack of regulation of ghrelin by leptin [14], the adipocyte-derived leptin and the gut-derived PYY systems act independently of each other. The question of whether leptin alters the response to PYY, as it does for the intestinal response to cholecystokinin in leptin-deficient mice [15], and/or whether more long-term administration of leptin alters either the PYY levels or the PYY response, requires further evaluation.

Short-term gut-derived appetite signals such as PYY and long-term adipostatic signals such as leptin may play different roles in the complex system of energy homeostasis that tightly defends body weight over the long term despite short-term variations in food intake. Hence, clarification of the interaction between such appetite-regulating signals is important for advancing our understanding of the mechanisms by which appetite and body weight are regulated in humans, especially since redundancy of systems that regulate body weight may contribute to the high tendency for weight regain following attempts at weight loss. These findings also have relevance for the potential development of PYY, which has recently undergone phase 1 safety and tolerability studies [16], as an agent for the treatment of obesity. In this context, a greater understanding of the mechanisms by which this hormone acts is important.

In summary, these findings indicate that PYY is regulated by caloric administration and restriction in a manner that is consistent with a hormone that decreases appetite, but shortterm regulation of PYY appears to be independent of the adipocyte-hormone leptin, indicating that these two pathways for regulating energy homeostasis may act independently and in parallel to each other. These findings, which increase our understanding of appetite-regulating signals, may have relevance for the future development of therapeutic agents for obesity. Further investigation is warranted to determine whether PYY $3-36$ replacement could represent an effective anti-obesity therapy.

Acknowledgements This study was supported by NIH Grant MO1-RR01032, NIH Grant R01-58785, an Amgen grant to C. S. Mantzoros and an NIH Grant K23 RR018860 to J. L. Chan. We gratefully thank the GCRC nurses at BIDMC for collecting the samples for this research, and the GCRC nutritionists for their help with the isocaloric and fasting diets.

Duality of Interest No financial disclosures or conflicts of interest are reported by the authors.

\section{References}

1. Batterham RL, Cowley MA, Small CJ et al (2002) Gut hormone PYY(3-36) physiologically inhibits food intake. Nature 418:650-654

2. Challis BG, Pinnock SB, Coll AP, Carter RN, Dickson SL, O'Rahilly S (2003) Acute effects of PYY3-36 on food intake and hypothalamic neuropeptide expression in the mouse. Biochem Biophys Res Commun 311:915-919

3. Batterham RL, Cohen MA, Ellis SM et al (2003) Inhibition of food intake in obese subjects by peptide YY3-36. N Engl J Med 349:941-948

4. Tschop M, Castaneda TR, Joost HG et al (2004) Physiology: does gut hormone PYY3-36 decrease food intake in rodents? Nature 430:1

5. Korner J, Bessler M, Cirilo LJ et al (2005) Effects of Roux-en$\mathrm{Y}$ gastric bypass surgery on fasting and postprandial concentrations of plasma ghrelin, peptide YY, and insulin. J Clin Endocrinol Metab 90:359-365

6. Mantzoros CS (1999) The role of leptin in human obesity and disease: a review of current evidence. Ann Intern Med 130: 671-680

7. Adrian TE, Ferri GL, Bacarese-Hamilton AJ, Fuessl HS, Polak JM, Bloom SR (1985) Human distribution and release of a putative new gut hormone, peptide YY. Gastroenterology 89: $1070-1077$

8. Chan JL, Heist K, DePaoli AM, Veldhuis JD, Mantzoros CS (2003) The role of falling leptin levels in the neuroendocrine and metabolic adaptation to short-term starvation in healthy men. J Clin Invest 111:1409-1421

9. Chan JL, Moschos SJ, Bullen J et al (2005) Recombinant methionyl human leptin administration activates signal transducer and activator of transcription 3 signaling in peripheral blood mononuclear cells in vivo and regulates soluble tumor necrosis factor- $\alpha$ receptor levels in humans with relative leptin deficiency. J Clin Endocrinol Metab 90: 1625-1631

10. Wong SL, DePaoli AM, Lee JH, Mantzoros CS (2004) Leptin hormonal kinetics in the fed state: effects of adiposity, age, and gender on endogenous leptin production and clearance rates. J Clin Endocrinol Metab 89:2672-2677

11. Alvarez BM, Borque M, Martinez-Sarmiento J et al (2002) Peptide YY secretion in morbidly obese patients before and after vertical banded gastroplasty. Obes Surg 12:324-327

12. Considine RV, Sinha MK, Heiman ML et al (1996) Serum immunoreactive-leptin concentrations in normal-weight and obese humans. N Engl J Med 334:292-295

13. Heymsfield SB, Greenberg AS, Fujioka K et al (1999) Recombinant leptin for weight loss in obese and lean adults: a randomized, controlled, dose-escalation trial. JAMA 282: $1568-1575$

14. Chan JL, Bullen J, Lee JH, Yiannakouris N, Mantzoros CS (2004) Ghrelin levels are not regulated by recombinant leptin administration and/or three days of fasting in healthy subjects. J Clin Endocrinol Metab 89:335-343

15. Kiely JM, Graewin SJ, Pitt HA, Swartz-Basile DA (2005) Leptin increases small intestinal response to cholecystokinin in leptin-deficient obese mice. J Surg Res 124:146-150

16. Troupin B, Lush C, Chen K et al (2005) A phase 1 study to evaluate the safety, tolerability, and pharmacokinetics of rising doses of AC162352 (synthetic human PYY3-36) in lean and obese subjects. Endocrine Society Meeting, 4-7 June, 2005, San Diego: oral presentation (Abstract) 